\title{
Sideband locking of a single-section semiconductor distributed-feedback laser in an optical phase-lock loop
}

\author{
Naresh Satyan, ${ }^{1, *}$ Arseny Vasilyev, ${ }^{1}$ Wei Liang, ${ }^{2}$ George Rakuljic, ${ }^{3}$ and Amnon Yariv ${ }^{1}$ \\ ${ }^{1}$ Department of Electrical Engineering, California Institute of Technology, 1200 E. California Boulevard 136-93, \\ Pasadena, California 91125, USA \\ ${ }^{2}$ Currently with OEwaves, Inc., 1010 East Union Street Pasadena, California 91106, USA \\ ${ }^{3}$ Telaris, Inc., 2118 Wilshire Boulevard \#238, Santa Monica, California 90403, USA \\ *Corresponding author: naresh@caltech.edu
}

Received July 8, 2009; revised September 18, 2009; accepted September 19, 2009; posted September 30, 2009 (Doc. ID 113927); published October 19, 2009

The bandwidth and performance of optical phase-lock loops (OPLLs) using single-section semiconductor lasers (SCLs) are severely limited by the nonuniform frequency modulation response of the lasers. It is demonstrated that this restriction is eliminated by the sideband locking of a single-section distributed-feedback SCL to a master laser in a heterodyne OPLL, thus enabling a delay-limited loop bandwidth. The lineshape of the phase-locked SCL output is characterized using a delayed self-heterodyne measurement. (C) 2009 Optical Society of America

OCIS codes: $140.5960,290.3700,030.1640$.

Semiconductor laser (SCL) optical phase-lock loops (OPLLs) have found applications in a number of fields, including microwave photonics, coherent optical communications, sensing, and coherent optical arrays [1-4]. In a typical heterodyne OPLL, depicted in Fig. 1, the SCL acts as a "slave" current controlled oscillator, whose frequency and phase are locked to a master laser offset by an rf offset signal. For stable loop operation, it is necessary that the loop bandwidth, defined as the bandwidth over which the phase error between the slave and the master lasers is corrected by the loop, be much larger than the summed linewidths of the two lasers.

Using a standard small signal analysis, the openloop transfer function of the OPLL shown in Fig. 1, with respect to the phase of the slave SCL, is given by [5]

$$
G(\omega)=\frac{K F_{F M}^{S C L}(\omega) F_{f}(\omega) F_{P D}(\omega) F_{M}(\omega) e^{-j \omega \tau_{d}}}{j \omega},
$$

where $K$ is the open-loop dc gain and $\tau_{d}$ is the loop propagation delay. $F_{F M}^{S C L}(\omega), F_{f}(\omega), F_{P D}(\omega)$, and $F_{M}(\omega)$ are the normalized transfer functions of the SCL FM response, loop filter, photodetector (PD), and rf mixer, respectively. The maximum achievable bandwidth of an OPLL is therefore ultimately limited by the loop propagation delay [6], and OPLLs constructed using free-space optics [1] or integrated optics [7] offer the possibility of achieving bandwidths up to a few gigahertz. However, a more stringent limitation on the loop bandwidth is imposed by the FM response $F_{F M}^{S C L}(\omega)$, particularly for single-section SCLs. The FM response of a single-section SCL exhibits a characteristic phase reversal in the frequency range of $0.5-5 \mathrm{MHz}$ [8], corresponding to a redshift with increasing current at lower modulation frequencies and a blueshift at higher frequencies. This limits the bandwidth of a negative-feedback system using the
SCL to a few megahertz. Consequently, it is not possible to achieve stable loop operation using standard distributed-feedback (DFB) SCLs with linewidths of a few megahertz. The availability of SCLs with suitable FM characteristics is therefore the biggest challenge in constructing stable OPLLs. While multielectrode SCLs have typically been used to obviate this problem, they do not offer the robustness and simplicity of operation of single-section DFB SCLs. Other approaches to overcome the thermal-induced bandwidth limitation have included the use of external-cavity SCLs with narrow linewidths [9] or the use of an additional optical injection locking loop [10]. In this Letter, we demonstrate that the limitation imposed by the phase reversal of the FM response of a single-section SCL can be eliminated using a sideband-locked heterodyne OPLL, thereby reducing system complexity and enabling delaylimited SCL-OPLLs using readily available SCLs.

The FM response of a single-section SCL is determined by a thermal redshift at low frequencies and an electronic blueshift at higher frequencies, leading to a dip in the amplitude response and a phase reversal at a few megahertz [8]. At frequencies between

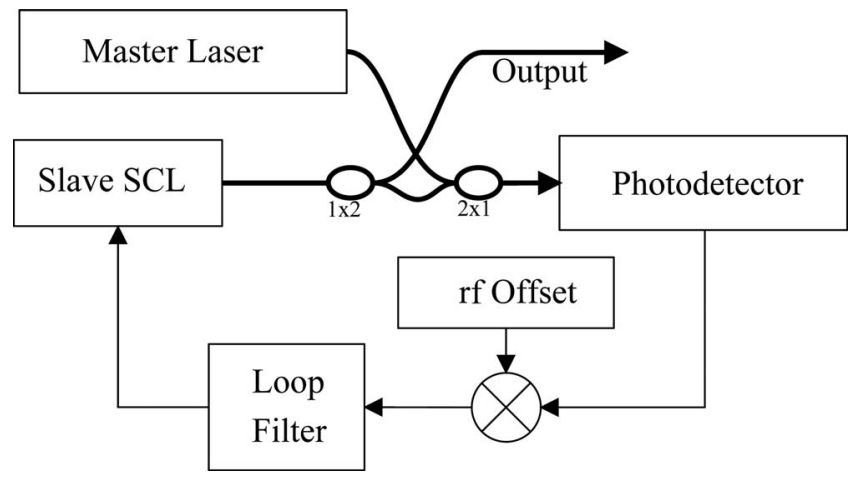

Fig. 1. Schematic diagram of a heterodyne OPLL. The optical path is denoted by thick lines. 
this crossover frequency and the relaxation resonance frequency of the laser $(\sim 10 \mathrm{GHz})$, the amplitude and phase of the FM response are constant. If the feedback current into the SCL is upshifted into this frequency range, a much wider frequency range is opened up for phase-locking, and loop bandwidths of up to a few gigahertz are achievable.

Consider the heterodyne sideband-locked OPLL system shown in Fig. 2. A part of the SCL output is combined with the master laser using a $2 \times 1$ fiber coupler, and mixed in a high-speed PD. The error signal at the output of the PD is mixed with an rf offset signal, filtered, and fed into a voltage-controlled oscillator (VCO). The VCO output is in turn fed into the SCL, creating multiple FM sidebands whose frequency and phase are given by $\omega_{S, n}=\omega_{S}+n \omega_{V}$ and $\phi_{S, n}=\phi_{S}+n \phi_{V}$, respectively. Any one of these sidebands can then be locked to the master laser. The frequency and phase of the phase-locked $n$th sideband are then given by $\omega_{S, n}^{\text {locked }}=\omega_{M}-\omega_{r f}$ and $\phi_{S, n}^{\text {locked }}=\phi_{M}$ $-\phi_{r f}$. The locked $n$th sideband is coherent with the master laser, while the other sidebands are necessarily incoherent and have to be optically filtered out. The power in the $n$th sideband (normalized to the total optical power) is given by

$$
P_{n}=\left|J_{n}\left(\frac{\left|F_{F M}^{S C L}\left(\omega_{V}\right)\right| A_{V}}{\omega_{V}}\right)\right|^{2},
$$

where $J_{n}$ is the $n$ th-order Bessel function of the first kind and $A_{V}$ is the amplitude of the modulating current at the VCO output. To maximize the total coherent power, the $n=1$ sideband is phase locked, and the amplitude $A_{V}$ is chosen so as to maximize the power in the first sideband. From Eq. (2), at the optimal value of $A_{V}, 33.6 \%$ of the total power is in the first sideband. This power penalty introduced by the filtering of the incoherent sidebands is acceptable in most applications of OPLLs owing to the high output power of the SCLs.

The open-loop transfer function of the system shown in Fig. 2, with respect to the phase of the first optical FM sideband is given by

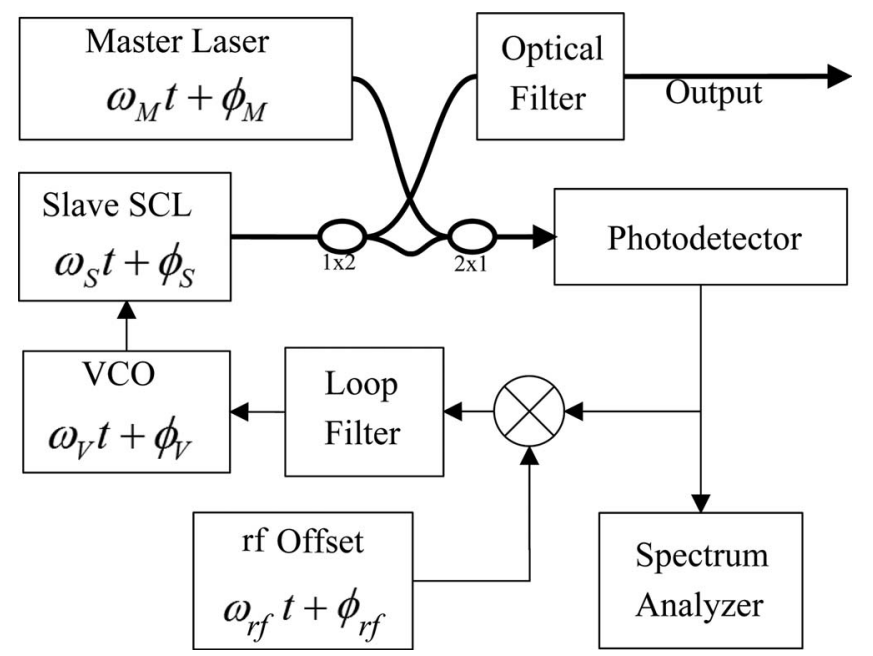

Fig. 2. Schematic diagram of a heterodyne sidebandlocked OPLL.

$$
G_{1}(\omega)=\frac{K_{1} F_{F M}^{V C O}(\omega) F_{f}(\omega) F_{P D}(\omega) F_{M}(\omega) e^{-j \omega \tau_{d}}}{j \omega}
$$

where $K_{1}$ is the open-loop dc gain and $F_{F M}^{V C O}(\omega)$ is the normalized FM response of the VCO. Equation (3) is valid whenever the nominal VCO frequency is chosen to be in the frequency range where the FM response of the SCL is constant. The loop bandwidth is therefore constrained by the FM bandwidth of the VCO and the loop propagation delay. If a high-bandwidth VCO is used, the loop bandwidth is limited primarily by the propagation delay in the loop.

The sideband-locking experiment was demonstrated using a commercially available fiber-coupled DFB SCL with an output power of $40 \mathrm{~mW}$ at $1550 \mathrm{~nm}$, and a tunable master laser with a linewidth of $\sim 50 \mathrm{kHz}$. The loop PD had a bandwidth of $12 \mathrm{GHz}$. The measured FM response of the SCL, shown in Fig. 3, exhibits a $\pi / 2$ phase crossover at a frequency of $1.6 \mathrm{MHz}$, which is less than its $3 \mathrm{~dB}$ linewidth of $5 \mathrm{MHz}$; and the SCL cannot therefore be phase locked in the simple heterodyne OPLL of Fig. 1. However, using the sideband-locking technique presented in this Letter, the first FM sideband of this SCL was successfully phase locked to the master laser in a fiber-based OPLL using discrete rf electronic components. The frequencies of the VCO and the rf offset signal were chosen to be $4 \mathrm{GHz}$ and $1.5 \mathrm{GHz}$, respectively. The locked FM sideband was optically filtered using a fiber Bragg grating with a narrow $20 \mathrm{~dB}$ bandwidth of $10 \mathrm{GHz}$. A suppression ratio of $>25 \mathrm{~dB}$ to the carrier and the $n=2$ sideband was achieved.

The bandwidth of the fiber-based OPLL without a loop filter was about $20 \mathrm{MHz}$, corresponding to a total loop propagation delay of $12.5 \mathrm{~ns}$. By varying the fiber delay in the loop, it was verified that the bandwidth was limited by the loop delay. A passive R-C filter with the transfer function

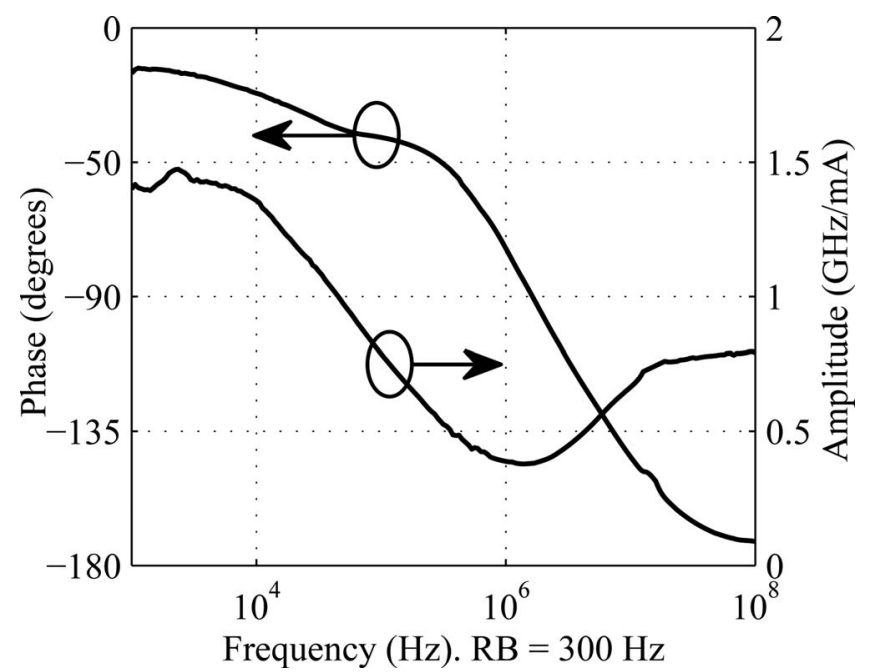

Fig. 3. Measured FM response of the DFB SCL. 


$$
F_{f}(\omega)=\frac{\left(1+j \omega \tau_{z 1}\right)\left(1+j \omega \tau_{z 2}\right)}{\left(1+j \omega \tau_{p 1}\right)\left(1+j \omega \tau_{p 2}\right)}
$$

was used in the loop to improve the bandwidth, with $\tau_{z 1}=53.6 \mathrm{~ns}, \quad \tau_{z 2}=1.41 \mu \mathrm{s}, \quad \tau_{p 1}=4.34 \mathrm{~ns}, \quad$ and $\tau_{p 2}$ $=8.71 \mu \mathrm{s}$. The resultant loop bandwidth was measured to be $35 \mathrm{MHz}$, and the hold-in range was $\pm 90 \mathrm{MHz}$. The measured spectrum of the beat signal between the phase-locked FM sideband and the master laser is shown in Fig. 4. The locking efficiency $\eta$, defined as the ratio of coherent power to the total power in the optical sideband, is calculated from the spectrum to be $80 \%$. This corresponds to a residual phase error $\sigma_{\phi}=28.6^{\circ}$, calculated using the relation $\sigma_{\phi}=\left(\eta^{-1}-1\right)^{1 / 2}[11]$. The loop bandwidth and the residual phase error can be further improved by reducing the loop propagation delay.

The lineshape of the master laser and that of the filtered $n=1$ sideband of the slave SCL, measured using a delayed self-heterodyne interferometer technique, are shown in Fig. 5. The lineshape of the locked SCL sideband follows that of the master laser for frequencies within the loop bandwidth, and reverts to the unlocked lineshape outside the loop bandwidth.

In summary, the limitation imposed on the loop bandwidth of an OPLL using a single-section DFB SCL by the phase reversal of the laser FM response can be overcome by locking an FM sideband of the SCL to the master laser. The sideband locking of a DFB laser, which could not be locked in a simple heterodyne OPLL, was experimentally demonstrated. A delay-limited bandwidth of $35 \mathrm{MHz}$ was achieved, which can be increased to a few hundreds of megahertz using miniature or integrated optics and integrated rf electronic circuits. The phase-locked side-

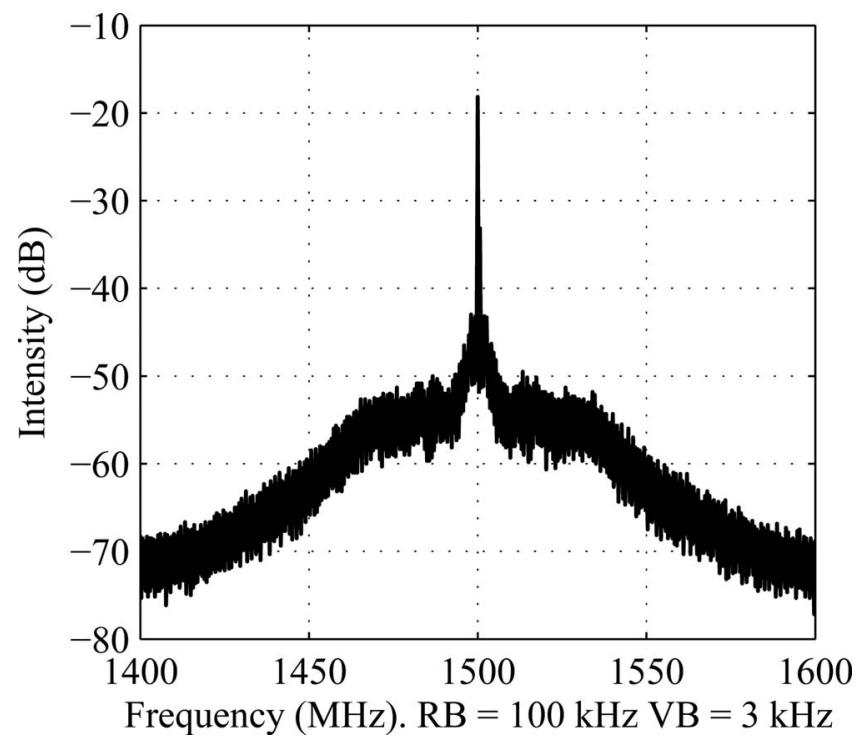

Fig. 4. Beat spectrum between the locked sideband and the master laser.

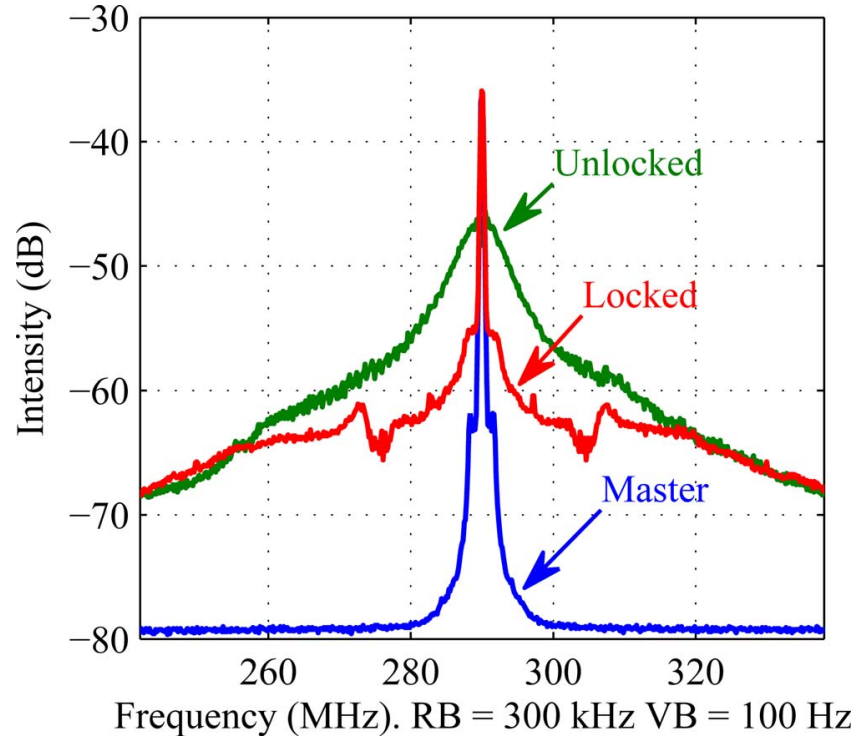

Fig. 5. (Color online) Lineshape measurements using a delayed self-heterodyne interferometer with a frequency shift of $290 \mathrm{MHz}$.

band was optically filtered, and it was shown that the phase noise of the filtered locked sideband was determined by that of the master laser for frequencies within the loop bandwidth. The demonstrated approach to phase-locking SCLs facilitates the use of standard single-section DFB SCLs in OPLLs with very little increase in system complexity.

The authors acknowledge the support of the Defense Advanced Research Projects Agency's (DARPA) Microsystems Technology Office (R. Esman).

\section{References}

1. L. Langley, M. Elkin, C. Edge, M. Wale, U. Gliese, X. Huang, and A. Seeds, IEEE Trans. Microwave Theory Tech. 47, 1257 (1999).

2. E. Ip, A. P. T. Lau, D. J. F. Barros, and J. M. Kahn, Opt. Express 16, 753 (2008).

3. N. Satyan, W. Liang, A. Kewitsch, G. Rakuljic, and A. Yariv, IEEE J. Sel. Top. Quantum Electron. 15, 240 (2009).

4. N. Satyan, W. Liang, and A. Yariv, IEEE J. Quantum Electron. 45, 755 (2009).

5. F. Gardner, Phaselock Techniques, 3rd ed. (Wiley, 2005).

6. R. Ramos and A. Seeds, Electron. Lett. 26, 389 (1990).

7. S. Ristic, A. Bhardwaj, M. J. Rodwell, L. A. Coldren, and L. A. Johansson, in Optical Fiber Communication Conference, OSA Technical Digest (CD) (Optical Society of America, 2009), paper PDPB3.

8. P. Corre, O. Girad, and I. F. de Faria, Jr., IEEE J. Quantum Electron. 30, 2485 (1994).

9. R. Steele, Electron. Lett. 19, 69 (1983).

10. A. Bordonalli, C. Walton, and A. Seeds, IEEE Photon. Technol. Lett. 8, 1217 (1996).

11. D. R. Stephens, Phase-Locked Loops for Wireless Communications: Digital and Analog Implementation (Kluwer Academic Publishers, 1998), Chap. 12. 